Mini Review

\title{
The Roles of Doxorubicin in Hepatocellular Carcinoma
}

\author{
Kin Tam \\ Faculty of Health Science, University of Macau, Macau, China \\ E-mail: kintam@umac.mo; Tel.: +853-8397-8501; Fax: +853-8397-8500 \\ Received: June $01^{\text {st }}, 2013$; Revised: July $03^{\text {rd }}, 2013$, Published: July $03^{\text {rd }}, 2013$
}

\begin{abstract}
Liver cancer is the third most common cause of death from cancer worldwide. Hepatocellular carcinoma (HCC) accounts for $80-90 \%$ of all primary liver cancers. The mortality of HCC is high, and the treatment options are limited. Doxorubicin exhibits a broad spectrum of anti-tumour activity. When administrated via the hepatic artery, doxorubicin showed anti-tumour effects and partial response in HCC patients. In this mini review, we will first outline the treatment options for HCC. Then, we will briefly discuss the pharmacological, toxicological and pharmacokinetic aspects of doxorubicin. Finally, we will review the new developments and future directions of using doxorubicin in the therapy of HCC.
\end{abstract}

\section{Keywords}

primary liver cancer; anticancer; cytotoxic drug; transarterial chemoembolization; drug eluting beads

\section{Introduction}

Liver cancer is the third most common cause of death from cancer worldwide. Globally, over 600,000 people develop liver cancer and the fatality rate is high [1]. The highest incidence of liver cancer occurs mostly in developing countries, such as in sub-Saharan Africa and Eastern Asia, and especially in men: the overall male: female sex ratio is about 2.4 [1]. Regions with low incidence include North and South America, Australia, and Northern Europe. Hepatocellular carcinoma (HCC) accounts for $80-90 \%$ of all primary liver cancers [2]. Patients with cirrhosis exhibit a high probability of developing HCC [3]. Many factors can lead to the development of cirrhosis. These include: infection with the hepatitis B or hepatitis C viruses, alcoholism, inherited metabolic diseases, diabetes and smoking as well as exposure to aflatoxins, which is a group of mycotoxins produced by the Aspergillus fungus in foodstuffs like corn and peanuts during storage in warm, damp conditions [4-7].

Doxorubicin was one of the early anthracyclines, isolated from Streptomyces peucetius almost four decades ago [8]. It has been regarded as one of the effective chemotherapy drugs for cancer treatment [9]. Unfortunately, the clinical uses of this drug are hampered by cumulative cardiotoxicity, with the risk becoming ever greater at cumulative doses $\geq 550 \mathrm{mg} / \mathrm{m}^{2}$ [10]. In HCC chemotherapy, clinical studies showed that systemic administration of doxorubicin showed limited clinical benefits. However, when the drug was given by the hepatic artery route, tumour shrinkage and partial responses were seen in $30-70 \%$ of patients, usually associated with some form of hepatic artery occluding agent [11]. 
There are ongoing intensive preclinical and clinical efforts to search for better drugs and treatment regimens for HCC. While the research continues, it is valuable to pursue a deeper understanding of old drugs like doxorubicin, with the broad aims of identifying and/or devising new strategies for its better use in patients. In this mini review, we will first outline the treatment options for HCC. Then, we will briefly discuss the pharmacological, toxicological and pharmacokinetic aspects of doxorubicin. Finally, we will review the development and future directions of using doxorubicin in therapy for HCC.

\section{Treatment options for hepatocellular carcinoma}

$\mathrm{HCC}$ is often diagnosed late in its course, partly because of the lack of distinctive pathological symptoms [12]. As a result, the prognosis is poor, with a median survival of $1-8$ months and a 5-year survival rate of only $3 \%$ [13]. Despite the progress in early diagnosis [14], the treatment options are limited. Hepatic resection and liver transplantation represent the curative treatment options, offering the prospect of cure with 5 -year survival rates of up to $50[15,16]$ and $70 \%[17,18]$, respectively.

However, only $10-20 \%$ of patients are suitable for surgical resection due to the burden of hepatic tumour, the presence of extrahepatic spread, or the extent of underlying liver disease. Liver transplantation is the only surgical option for HCC patients with impaired liver function. In view of the severe shortage of liver donors, hepatic resection is still regarded as the mainstay of therapy in patients with preserved hepatic function. For non-surgical patients with early stage HCC, ablative techniques could be beneficial. Commonly used liver-directed therapies include percutaneous ethanol injection and radiofrequency ablation (see also section 5.3). Other investigative techniques include percutaneous acetic acid injection [19], microwave coagulation [20], cryoablation [21], and laser thermotherapy [22].

Chemotherapy is often used to slow the progress of advanced liver cancer [23]. Typically, this involves systemic administration (either intravenous or oral) of anti-cancer drugs such as doxorubicin, 5-fluorouracil, or cisplatin. Even these drugs shrink only a small portion of tumours and the responses often do not last long. In particular, the side effects and cardiotoxicity of doxorubicin can limit its routine use at an efficacious dose. Targeted chemotherapy, represented by newer drugs such as sorafenib, has been shown to slow the growth of advanced liver cancer and exhibit less severe side effects [24]. Sorafenib is a potent multikinase inhibitor with antiangiogenic and antiproliferative properties. It targets the serine-threonine kinases Raf- 1 and B-Raf, the tyrosine kinases of vascular endothelial growth factor receptors (VEGFR-1/2/3) as well as the platelet-derived growth factor receptor $\beta$ (PDGFR- $\beta$ ). Cellular signalling in connection with Raf-1 and VEGF plays an important role in the development of HCC [25]. With this new treatment, median survival and time to radiologic progression were extended by about 3 months for patients with advanced HCC [25].

Over the years, clinical studies have been carried out to broaden the treatment options. These include radioembolotherapy, adjuvant therapy, and intra-arterial approaches [26,27]. Palliative therapies via transarterial chemoembolization (TACE) are used for liver cancer not amenable to surgical procedures $[28,29]$. In the next section (2.1), we will briefly discuss the TACE procedure.

\subsection{Transarterial chemoembolization (TACE)}

Figure 1 shows a schematic diagram of a TACE procedure, which involves combined intra-arterial administration of cytotoxic anticancer drug(s) and embolization agent(s) into a liver tumour. 

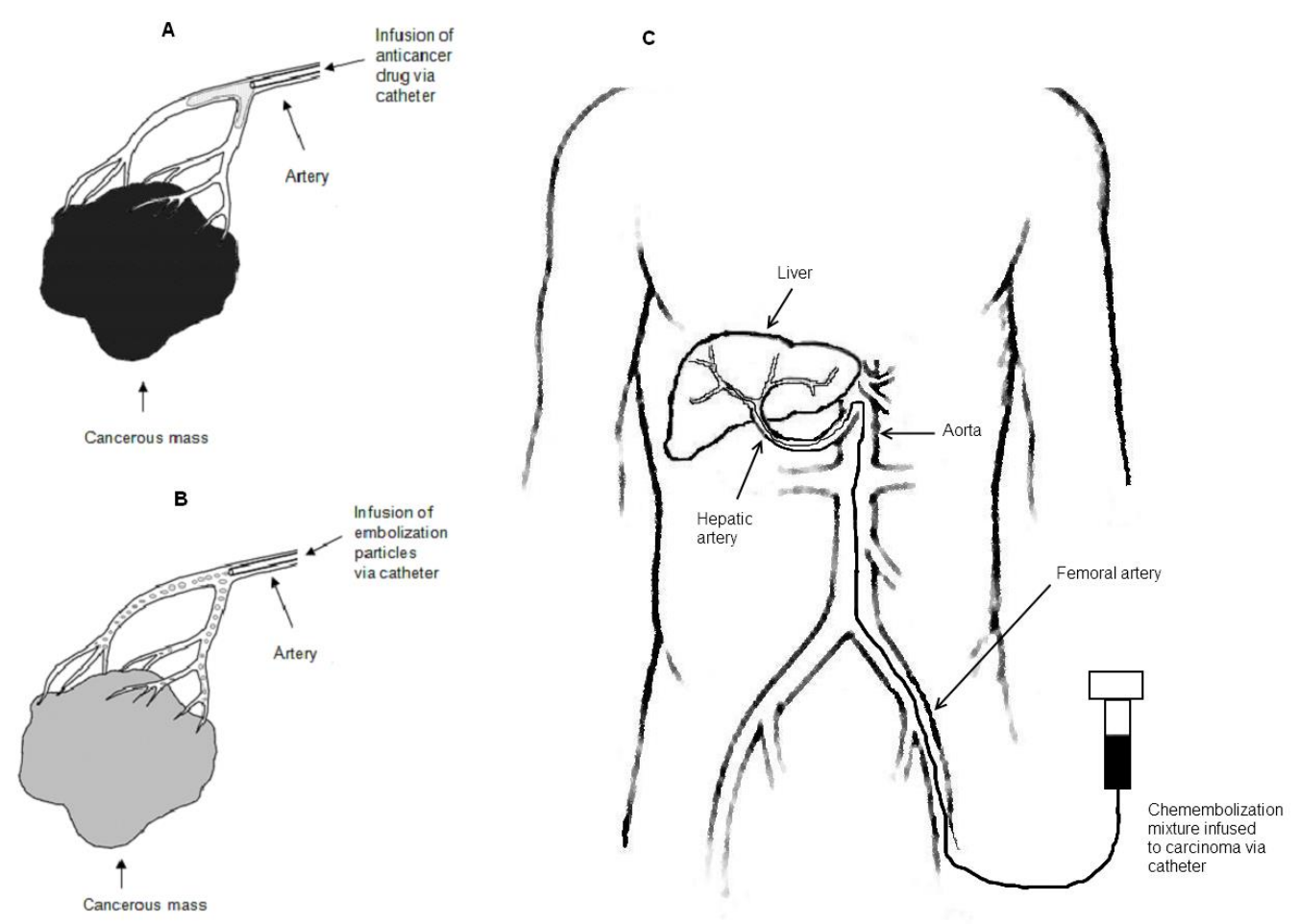

Figure 1. Schematic diagrams show: $(\boldsymbol{A})$ targeted intra-arterial infusion of anti-cancer drugs leading to high local concentration at the cancerous mass, $(B)$ the infusion of embolization particles leading to ischemia of the cancerous mass, and $(C)$ the intra-arterial infusion procedure which involves placement of a catheter through the femoral artery into a branch of the hepatic artery (anti-cancer drugs, embolization particles, or drug eluting beads can be delivered to the cancerous mass).

A multidisciplinary team consisting of a surgeon, clinical oncologist and radiologist in a hospital usually conduct the procedure. There are variants on these transarterial techniques, which are dependent on the experience of the clinical specialists and the particular treatment protocols of the hospital. The simplest form of transarterial chemoembolization (TACE) involves a 2-step process, namely, (1) intra-arterial administration of chemotherapeutic agents into the tumour-feeding artery via an intra-arterially inserted catheter, and (2) selective embolization of the tumour-feeding artery. Some investigators refer to this approach as conventional TACE. The liver has a unique dual blood supply from both the portal vein and the hepatic artery. The normal parenchyma of the liver receives two-thirds of its necessary blood supply from the portal vein and receives the remaining one-third from the hepatic artery. Hypervascular tumours such as hepatocellular carcinomas (HCCS) receive their blood supply mainly from the hepatic artery $[30,31]$. Thus, intra-arterial infusion of a cytotoxic anticancer drug (see section 2.2) with a viscous oily material, lipiodol, followed by embolization of the artery with embolic agent(s) which themselves will occlude the arterial blood supply to the tumour, cause an infarct and subsequent necrosis of the tumour. Embolic agents commonly used in TACE procedure include gelatin sponge, polyvinyl alcohol (PVA) particles, and degradable starch microspheres.

TACE treatment for liver malignance can induce extensive tumour necrosis in most patients. However, the ability of TACE to induce tumour necrosis does not necessarily mean longer survival rates for HCC patients. Almost $70-80 \%$ of patients treated with TACE die due to tumour progression during follow-up because of the eventual re-growth of residual tumour cells after regaining a vascular supply or remote recurrence of tumours in other areas of the liver [32]. TACE is not indicated for curative therapies. In 2002, two randomized controlled trials (RCTs) from Hong Kong [33] and Spain [34] showed the survival benefits of TACE compared to the best conservative treatment. These RCTs were followed by cumulative meta- 
analyses that included all published RCTs [35,36], showing that TACE significantly reduced the overall 2-year mortality rate compared to control patients who received conservative or inactive treatments.

\subsection{Cytotoxic anticancer drugs}

In TACE, the most common cytotoxic drugs are doxorubicin, followed by cisplatin, epi/doxorubicin, mitoxantrone and mitomycin $\mathrm{C}$. The dose of the cytotoxic drug given depends on the size of the tumour, the position of the catheter, the patient's liver function, and the response to previous courses of TACE, if any. However, the criteria for determining the dosages of chemotherapeutic agents are variable and not standardized: some clinicians prefer to factor in the patient's body surface area, weight, tumour burden or bilirubin level, while others use a fixed dose. In some early studies, 40 - $100 \mathrm{mg}$ of doxorubicin [37] or 10 $70 \mathrm{mg}$ of cisplatin [38] per session was used. A few randomized controlled trials (RCTs) failed to show significant differences in survival between the use of doxorubicin and other drugs such as cisplatin or epirubicin and, to date, there is no evidence of the superiority of any single chemotherapeutic agent over other drugs or for mono-drug chemotherapy versus combination chemotherapy. In the United States, the most common combination of chemotherapeutic agents includes a mixture of $100 \mathrm{mg}$ cisplatin, $50 \mathrm{mg}$ doxorubicin and $10 \mathrm{mg}$ mitomycin $\mathrm{C}$. It should be noted that some of the commonly used cytotoxic drugs in TACE, such as doxorubicin, could be infused directly into the systemic circulation for cancer chemotherapy but patients who receive this treatment may suffer from serious side effects (e.g. cardiac toxicity) [10]. Moreover, efficacy could be compromised by suboptimal drug concentration at the liver, since the dose distribution is mainly achieved via the systemic circulation. Indeed, the local drug concentration could be further limited by the fact that doxorubicin cannot diffuse very far through tissue that is necrotic, and the drug is not carried to the site when the neighbouring microvasculature is destroyed [39].

\subsection{Drug eluting beads}

More recently, advances have been made in materials design, such that the embolic agent itself can also be a drug carrier, which appears to be a more convenient and efficient procedure. Specifically, these drugloaded carriers are directly injected intra-arterially for the treatment of liver cancer in a single operation. This kind of drug carrier is referred to as a drug-eluting bead (DEB) in the literature. A typical example includes the DC Bead (Biocompatibles, Surrey, UK), which has undergone clinical investigations $[40,41]$. DC Beads are indicated for the treatment of a variety of malignant hypervascularised tumours, including HCC. It is a PVA based microspherical embolization agent, prepared from $\mathrm{N}$-acrylamidoacetaldehyde derivatized polyvinyl alcohol copolymerized with 2-acrylamido-2-methylpropane sulfonate. The presence of the anionic sulphonate group enables the sequestering of positively charged drugs, such as doxorubicin, epirubicin or irinotecan, by Coulomb charge interactions. The drug is slowly released from the beads at the site of administration. In particular, the interventional procedure is greatly simplified as the drug (e.g. doxorubicin) and the embolizing device (the sulfonate-modified PVA bead) are delivered the same time, which greatly facilitates the local and sustained delivery of the drug.

DC Beads are generally supplied in a hydrated form in a saline solution of suitable ionic strength in a vial. Before performing embolization, the saline solution is removed and the drug solution at the suggested concentration is mixed with the beads and left for an appropriate time, depending on the drug, the loading solution concentration and the bead size. Typically, loading will require a minimum of 20 minutes for the smallest bead size and up to 120 minutes for the largest. The beads are available in different size ranges: $100-300 \mu \mathrm{m}, 300-500 \mu \mathrm{m}, 500-700 \mu \mathrm{m}$, and $700-900 \mu \mathrm{m}$. Different drug loadings, varying from 5 $\mathrm{mg} / \mathrm{mL}$ to $45 \mathrm{mg} / \mathrm{mL}$ of hydrated beads, have been reported [42]. The recommended maximum dose of doxorubicin administered per treatment is $150 \mathrm{mg}\left(75 \mathrm{mg} / \mathrm{m}^{2}\right)$, with a maximum recommended lifetime 
dose of $450 \mathrm{mg} / \mathrm{m}^{2}$ in light of cardiac toxicity induced by the drug when it is introduced systemically [43]. Drug-loaded beads can be aspirated directly for the catheter delivery procedure. Patients may receive three or four chemoembolization treatments within 6 months. It has been demonstrated that DC Bead spheres can easily be loaded with doxorubicin to a recommended level of $25 \mathrm{mg} / \mathrm{mL}$ hydrated beads [42].

Lammer et al. reported a clinical study comparing DEB-TACE (intra-arterial injection of DC Beads loaded with doxorubicin) with conventional TACE (intra-arterial injection of doxorubicin in lipiodol followed by particle embolization with an embolic agent, such as PVA particles) for the treatment of cirrhotic patients with HCC [44]. The patient group treated with DEB-TACE showed higher response rates than the group treated with conventional TACE. Despite the hypothesis of superiority not being met, the clinical data suggested that the drug eluting bead treatment offered improved tolerance, with a significant reduction in serious liver toxicity, and a significantly lower rate of doxorubicin-related side effects [44]. In a more recent clinical study [45], Malagari reported the meta-analysis on the 5-year survival of HCC patients treated with DEB-TACE. It has demonstrated good responses, with overall survival rates of $93.6,62$, and $22.5 \%$ at 1,3 , and 5 years after sequential sessions of DEB-TACE in HCC patients not amenable to curative treatments. Thus, DEB-TACE appears to be safe and effective in the treatment of HCC and could offer a benefit to patients.

Regardless of the means of implementation, TACE is able to offer highly concentrated doses of chemotherapy for effective delivery to the tumour bed, while sparing the surrounding hepatic parenchyma [46]. Moreover, particle embolization of the tumour-feeding arteries sustains the effects of the chemotherapy by reducing its washout, allowing prolonged contact with the tumour. As a result, selective arterial obstruction-induced ischemic tumour necrosis is achievable while minimizing any damage to normal liver tissue, as the blood supply to the normal liver tissue is still maintained by the dominant blood flow from the portal vein. This combination of embolotherapy and regional chemotherapy has synergistic, anti-tumour effects, resulting in a high objective response rate.

In next two sections, we will turn our attention to the pharmacology, toxicity and pharmacokinetics of doxorubicin.

\section{Pharmacology and toxicity of doxorubicin}

Doxorubicin is a cytotoxic anthracycline antibiotic isolated from cultures of Streptomyces peucetius. It consists of a naphthacenequinone linked through a glycosidic bond at ring atom 7 to an amino sugar, daunosamine, with the structural formula shown in Fig. 2.

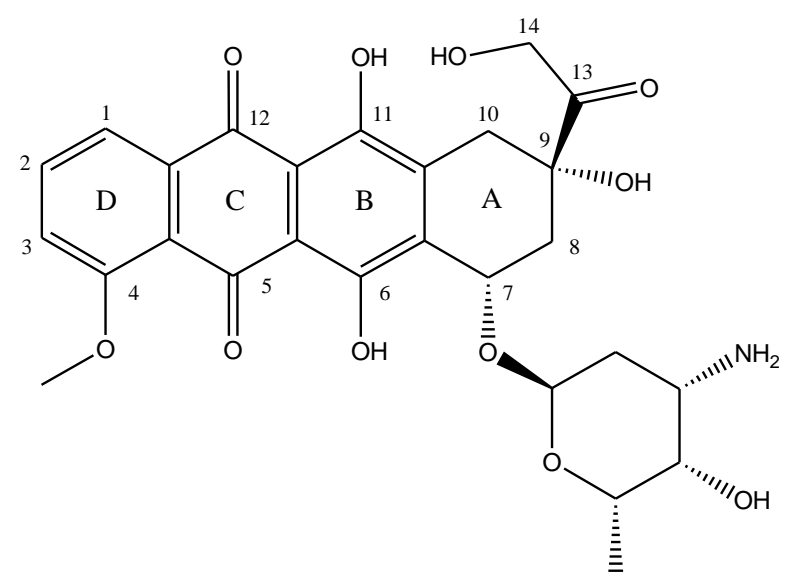

Figure 2. Structure of doxorubicin. 
The tetracyclic ring system is lipophilic, but the saturated end of the ring system contains several groups and the amino sugar, which forms a hydrophilic centre. The molecule itself is amphoteric, containing acidic functions in the phenolic groups and a basic function in the sugar amino group.

Doxorubicin is now widely used in the treatment of several of the most commonly diagnosed malignancies including leukaemia, lymphoma, bronchogenic, and traditional cell bladder carcinoma, as well as in the treatment of Wilms' tumour, neuroblastoma, sarcoma, and carcinoma of the breast, ovary, and stomach [47]. Liposomal doxorubicin formulations (Doxil ${ }^{\circledR}$ or Lipodox ${ }^{\circledR}$ ) have been developed to improve the therapeutic index of doxorubicin for use in conventional chemotherapy (systemic administration) while maintaining its anti-tumour activity. These liposomal formulations have been used for the treatment of metastatic breast/ovary cancers and of AIDS related Kaposi's Sarcoma [48]. Doxorubicin in a heat sensitive liposome formulation has been given an Orphan designation (EU/3/10/833) in Europe for the treatment of hepatocellular carcinoma [49].

Despite extensive clinical utilization, the exact mechanisms of action of doxorubicin in cancer cells remain a matter of controversy. For instance, doxorubicin has been reported to have at least seven different means of producing cellular dysfunction or death [50]. It is generally believed that the cytotoxic effects of doxorubicin on cancer cells are related to nucleotide base intercalation. In particular, the drug molecule stabilizes the topoisomerase II complex after it has broken the DNA chain for replication, preventing the DNA double helix from being resealed and thereby stopping the process of replication. Crystallographic data [51] suggest that the planar tetracyclic portion of the molecule intercalates between two base pairs of the DNA, while the sugar amino group sits in the minor groove and interacts with flanking base pairs immediately adjacent to the intercalation site. Moreover, it has been suggested that doxorubicin activates p53-DNA binding, leading to induced apoptosis [52,53]. Doxorubicin-induced apoptosis may result in therapeutic effects and/or toxicities.

Doxorubicin may undergo a cascade of free radical processes in normal and cancer cells. Typically this involves interactions of the molecule with the cell's electron transport chain, specifically the cytochrome P450 reductase (P450R) [54,55]. As shown in Fig. 3, the quinone moiety readily accepts the transfer of a single electron to form the semiquinone free radical, which could be directly cytotoxic in hypoxic environments via covalent modification of cellular macromolecules. Under aerobic conditions, the semiquinone radicals can shuttle electrons to molecular oxygen, giving rise to superoxide anion radicals [56-59]. These toxic intracellular radicals can be further converted to hydrogen peroxide and hydroxyl radicals, which can directly damage DNA, RNA, lipids, and proteins $[60,61]$. This oxidative stress mechanism appears to be a contributor to doxorubicin's ability to cause cell death, as well as its dose-limiting cardiotoxicity. In the process of electron shuttling, the doxorubicin ring system is restored to its original state and can be available for additional redox cycling reactions. 


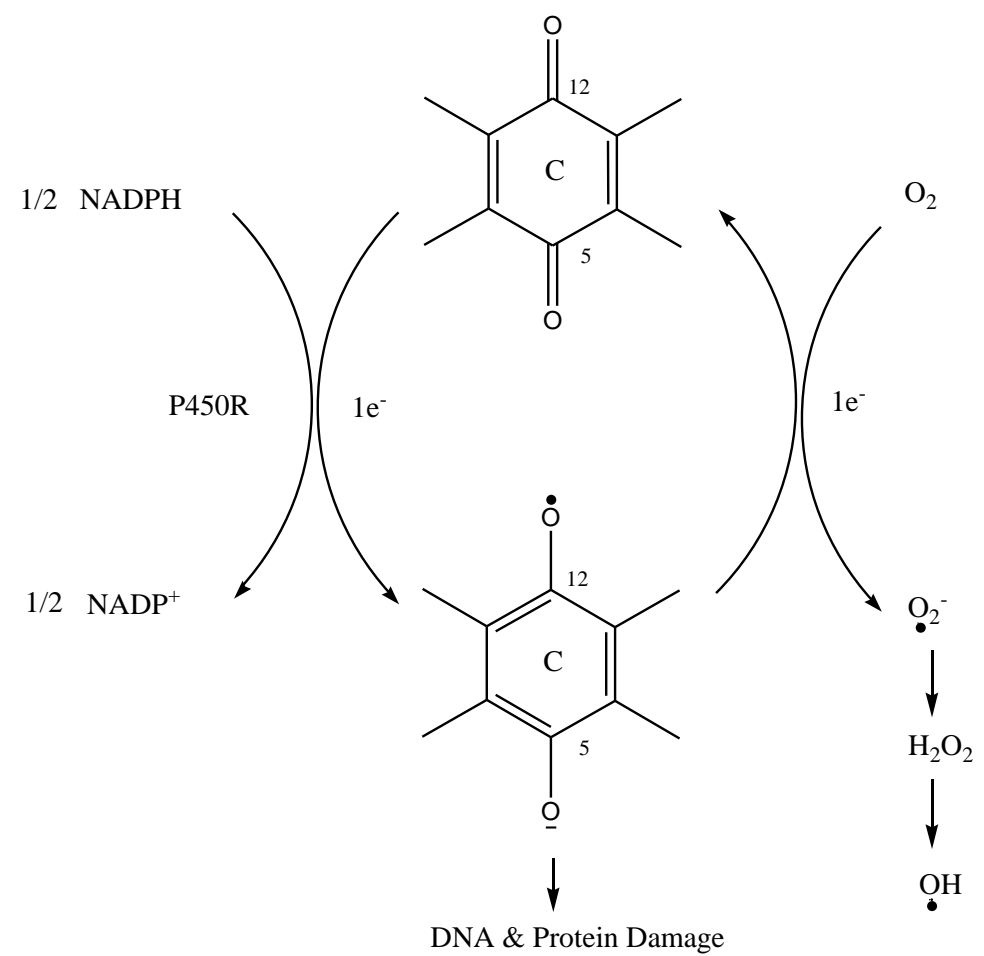

Figure 3. Simplified schematic of the redox cycling of doxorubicin.

The major doxorubicin metabolite in humans is doxorubicinol, which is produced via the cytosolic carbonyl reductase-catalyzed reduction of the ketone at C-13 on the parent drug (Fig. 2) [62,63]. Reductive deglycosylation at C-7 by P450R leads to formation of the 7-deoxy-doxorubicinol metabolite. Pharmacokinetic studies in humans revealed that doxorubicinol and 7-deoxy-doxorubicinol were the metabolites detected in vivo [64]. Doxorubicinol also exhibits antiproliferative and antineoplastic properties. Because doxorubicinol is more polar than the parent drug molecule, it is less likely to traverse through the cell membrane back to the extracellular space. Hence, it is more likely to stay inside cells, increasing its cytotoxic effects $[65,66]$. 7-deoxy-doxorubicinol is considerably less potent in inhibiting tumour growth in a mouse lymphocytic leukaemia cell line [67]. It has been suggested that doxorubicinol could accumulate in the heart and contribute significantly to the chronic cumulative cardiotoxicity of doxorubicin therapy [68].

Three types of toxicities are recognized for doxorubicin, namely acute, chronic, and local. The acute toxicities including nausea, vomiting, blood count suppression, and stomatitis are usually dose limiting. Also, alopecia occurs in more than half of patients receiving standard intermittent doses [9]. Congestive heart failure and dilated cardiomyopathy are chronic toxicities of doxorubicin that have received considerable attention [10] and generated much worry, especially when patients have received large cumulative dosages. Pathologic findings from patients' endomyocardial biopsies revealed loss of myofibrils, dilation of the sarcoplasmic reticulum, vascular degeneration, swelling of mitochondria, and increased numbers of lysosomes. This morphologic pattern has also been seen in rodents (e.g. mice and rats) dosed with doxorubicin, suggesting the existence of a species-independent pathway leading to these morphologic changes [69]. An early clinical study demonstrated a $10 \%$ incidence of clinical cardiomyopathy at a cumulative doxorubicin dosage of $550 \mathrm{mg} / \mathrm{m}^{2}$, with a sharp increase in the incidence curve at progressively higher dosages [10]. However, it has been reported that the probability of clinical congestive heart failure is never zero with any dose of doxorubicin, with some patients developing this complication even after a single dose [70]. Long-term follow-up is usually necessary because congestive heart failure may develop several years after therapy is completed. Although the mechanisms leading to the cardiotoxicity are not 
fully understood, these are thought to related to the activities of toxic metabolites, particularly doxorubicinol, and to the generation of reactive oxygen species within the cardiomyocytes (see Fig. 3). The most common hypothesis of doxorubicin-induced cardiotoxicity is the oxidative stress theory, where the principal toxic mechanisms involve iron and redox reactions [71]. It has been reported that when cardiomyocytes are exposed to doxorubicin, activation of the nuclear transcription factor NFkB and apoptosis occurred after redox cycling and formation of superoxide anion radicals and hydrogen peroxides [72]. Moreover, it has been shown that iron (Fe(II)) plays a key role in lipid peroxidation [73]. Because cells have very little or no free iron available to catalyse free radical reactions [74], it is conceivable that doxorubicin and doxorubicinol mediate iron release from ferritin and other cellular stores $[73,75]$. Toxic effects on a number of mitochondrial functions have been reported for metabolites of doxorubicin [76,77].

Two local toxicities of doxorubicin with potentially devastating consequences are extravasation skin injury (necrosis) [78] and the radiation recall reaction [79]. The development of extravasation skin injury in connection with doxorubicin is largely related to the therapy being given by intravenous infusion. With this route of administration, tissue damage can occur in close proximity to the infusion site, probably due to the drug is being absorbed by local cells in the tissue leading to cell death. The radiation recall reaction, which remains a poorly understood phenomenon, occurred in patients who had had prior irradiation for neoplastic disease. The observed reactions were painful, erythematous, and warm dermatitides located precisely in the previous field of irradiation.

\section{Pharmacokinetics of doxorubicin}

\subsection{Systemic administration}

Doxorubicin exhibits linear pharmacokinetics after intravenous administration. It is widely distributed in the plasma and tissues, with a volume of distribution exceeding $500 \mathrm{~L} / \mathrm{m}^{2}$. It undergoes triphasic plasma decay, with an initial half-life $\left(\mathrm{t}_{1 / 2 \alpha}\right)$ of $4.8 \mathrm{~min}$, a $\mathrm{t}_{1 / 2 \beta}$ of 2.6 hours and a terminal $\mathrm{t}_{1 / 2 \gamma}$ of about 48 hours [80]. In patients with normal liver function, hepatic clearance is high and more than $50 \%$ of the drug is excreted in the bile within 7 days after treatment. Due to the important role of hepatic metabolism and biliary excretion, patients with liver dysfunction show a rather different pattern in drug distribution. In particular, the half-life and AUC were found to be increased almost by 3-fold (and clearance decreased by almost 3fold) compared with patients with normal hepatic function [81].

On the other hand, the pegylated liposomal formulations of doxorubicin showed somewhat different pharmacokinetic performance. The presence of the outer polyethylene glycol polymer layer greatly reduces uptake of the drug by the reticulo-endothelial system, resulting in prolonged circulation in the plasma, relatively little tissue distribution and reduced volume of distribution. The pharmacokinetics are reported to be biphasic, with half-lives of $1-3$ hours and $30-90$ hours, respectively. The AUC after a dose of 50 $\mathrm{mg} / \mathrm{m}^{2}$ is approximately 300 -fold greater than that with free drug [82]. Clearance is drastically reduced (250-fold). Tumour neovasculature has been reported to permit penetration of liposomal doxorubicin into tumour tissue [82]. It has been shown that in a preclinical model, the hepatic distribution of liposomal doxorubicin was restricted to the vascular space and the liposomes were unable to pass through the fenestrations in the sinusoidal endothelium of the liver [83]. This observation may offer insight into the reduced hepatic metabolism of liposomal doxorubicin, since the drug is unable to gain access to the hepatocytes.

In HCC patients, it has been shown that when doxorubicin is dosed intra-arterially (without any subsequent embolization procedure), the pharmacokinetics is very similar to that of the intravenously 
dosed drug [84]. In other words, the drug disappearance profiles obtained from intra-arterial and intravenous administrations were similar. Clearly, intravenous or intra-arterial administration of doxorubicin alone may not necessarily be able to deliver a sufficient drug concentration to a hepatic tumour.

\subsection{DEB and locoregional administration}

Next, we turn our attention to DEB, namely the DC Beads loaded with doxorubicin. The in vitro elution kinetics of doxorubicin from DC Beads has been assessed using a T-cell apparatus, with a schematic depicted in Fig. 4 [42].

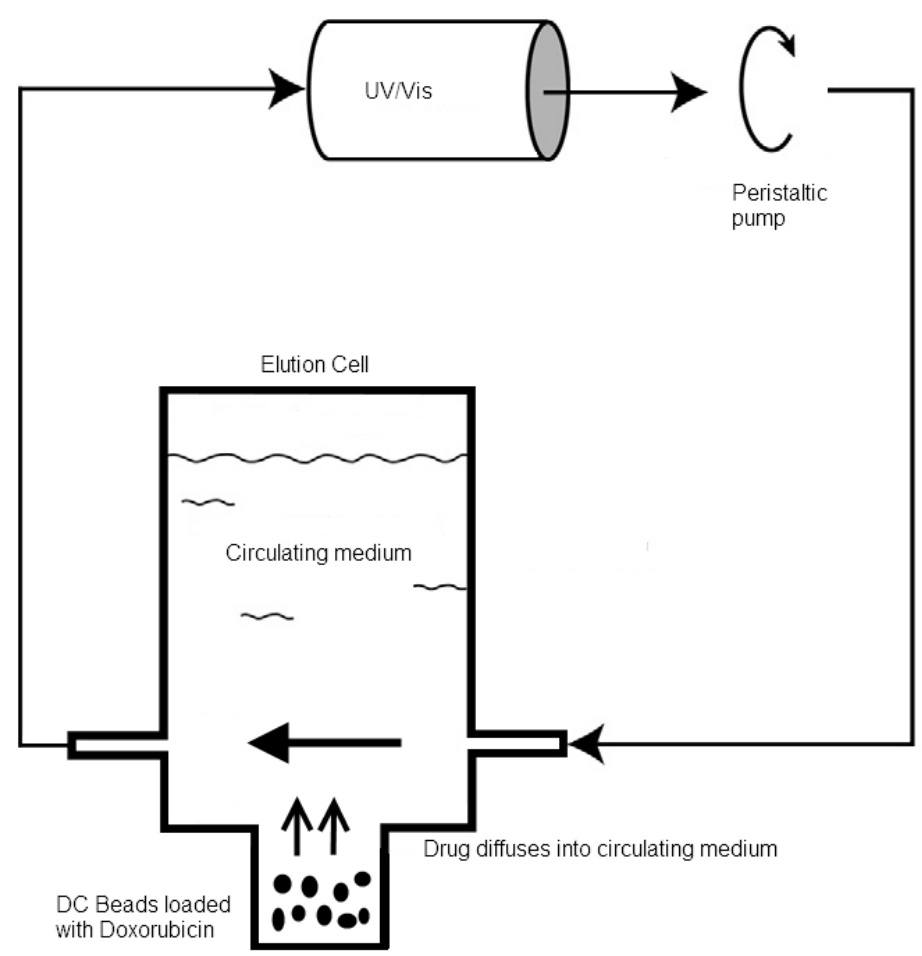

Figure 4. Schematic diagram of the T-cell apparatus (citation see text).

It has been shown that the doxorubicin did not release from the beads when the elution medium was pure water. When the elution medium contained ions and phosphate-buffered saline solution, reproducible and sustained release profiles were achievable. With a drug load of $25 \mathrm{mg} / \mathrm{mL}$ bead, it has been shown that the rate of drug release from $700-900 \mu \mathrm{m}$ beads was slower than that from $100-300 \mu \mathrm{m}$ beads, with half-lives of 1,730 and 150 hours, respectively [42]. These half-life data translate to less than $1 \%$ and $20 \%$ of drug being released over 24 hours from the total available drug loaded on the $700-900 \mu \mathrm{m}$ beads and $100-300 \mu \mathrm{m}$ beads, respectively. These in vitro data nicely demonstrated a sustained and controlled delivery of the doxorubicin without any burst effect, which may otherwise lead to acute toxicity in vivo. In a subsequent study [85], it was shown that the loading and release of doxorubicin followed a dose-response curve. Using $500-700 \mu \mathrm{m}$ beads, it was found that the half-live increased from 381 hours to 3658 hours as the concentration of the doxorubicin load increased from $6.25 \mathrm{mg} / \mathrm{mL}$ to $37.5 \mathrm{mg} / \mathrm{mL}$. For a fixed drug load of $37.5 \mathrm{mg} / \mathrm{mL}$, the half-life was only weakly dependent on bead size, with a minimum of 1505 hours for the $100-300 \mu \mathrm{m}$ beads. Interestingly, the in vitro elution data of doxorubicin has been shown to correlate well with the areas under the curve (i.e. the doxorubicin concentration in human plasma measured as a function of time) of 15 patients treated with DC Beads loaded with doxorubicin in the PRECISION clinical study [85]. This covered all doses used in the study: $6.25 \mathrm{mg} / \mathrm{mL}, 12.5 \mathrm{mg} / \mathrm{mL}, 18.75 \mathrm{mg} / \mathrm{mL}, 25 \mathrm{mg} / \mathrm{mL}$, and $37.5 \mathrm{mg} / \mathrm{mL}$ in 24 hours. As far as we are aware, this is the first report available in open literature to 
demonstrate a good in-vivo in-vitro correlation (IVIVC) for DC Beads. Undoubtedly, this kind of good IVIVC should help predict the local dose that should be administered before systemic effects (e.g. acute toxicity) occur. However, the amount of drug released in situ into tumour tissue in humans is difficult to determine and model by this kind of in vitro measure, because of practical and ethical issues. Studies on pre-clinical species using a $V x-2$ tumour rabbit model have been performed, and confirmed a high level of doxorubicin in the tumour over the entire period of study (14 days) associated with widespread necrosis of the tumour tissue [86].

The pharmacokinetics of doxorubicin in HCC patients have been determined as part of two phase I/II studies to evaluate the use of DC Beads in the DEB-TACE procedure. One of the studies compared the pharmacokinetic profiles of doxorubicin after injection of DC Beads against those seen after intra-arterial injection [87]. The average AUC after DC Bead injection was found to be about 100 -fold lower than intraarterial injection, despite a higher drug load being used in the DC Beads. The other study compared the pharmacokinetic profile of DEB-TACE against conventional TACE [88]. The peak drug concentration $\left(C_{\max }\right)$ was reached within 5 min after injection, but it was about 10-fold higher in conventional TACE patients. The AUC was also about 3-fold higher in the conventional TACE patients. It is evident that DEB-TACE is effective in limiting the systemic exposure to doxorubicin, resulting in reduced systemic toxicity.

The hepatic distribution of doxorubicin in HCC patients has recently been reported [89]. In this reported study, $6 \mathrm{HCC}$ patients were given the same DEB-TACE procedure followed by liver transplantation after embolization from 8 hours to 36 days. From liver explant samples, it was determined that the mean doxorubicin concentration surrounding the DEBs peaked at $5 \mu \mathrm{M}$ at 8 hours, and then diminished to 2.10 $\mu \mathrm{M}$ at 2 weeks, and $0.65 \mu \mathrm{M}$ at $32-36$ days. Most of the DC Beads $(100-300 \mu \mathrm{m})$ were confined within 10 $\mathrm{mm}$ of the tumour boundary. Necrosis of tissues surrounding DEBs has been observed in $9-36$ day liver explants, suggesting DEB may lead to antiproliferative and cytotoxic effects on the tumour cells surrounding the beads.

\section{The new developments and future directions}

HCC mortality is generally high and the available treatment options are limited. Chemotherapy using anti-tumour cytotoxic agents, such as doxorubicin, appears to be one of the non-curative therapeutic approaches to help HCC patients. Systemic chemotherapies have been evaluated for many years [11]. The response rate is poor (below $30 \%$ ) and the survival advantage is minimal. The uses of adjuvant or neoadjuvant systemic chemotherapy for liver resection also failed to show clinical benefits [11]. On the other hand, locoregional administration of doxorubicin via DEB-TACE produced relatively high response rates and survival advantage [45]. It would be particularly valuable to explore the best use of this approach in combination with other treatment options in the therapy of HCC.

\subsection{Standardization of TACE procedure}

One of the current issues with TACE concerns the technique of chemoembolization. Despite DEB offering some advantage, conventional TACE is still being used widely. While recommendations have been published by a panel of experts on the use of DEB-TACE [90], there is still no consensus on how to perform conventional TACE. For instance, there are variants on how the anticancer drug is formulated in Lipiodol. There is also a wide range of choices for embolizing agents [91,92]. Consequently, implementation could vary depending on the preference of the investigators. The evolution from conventional TACE towards DEBTACE will hopefully help to harmonize the implementation procedure. 


\subsection{Combination with targeted chemotherapy}

Targeted chemotherapy such as sorafenib represents a different mechanism of action against HCC by acting on Raf-1/VEGF cell signalling pathways. Used as a single agent, sorafenib can produce tumour shrinkage and offer a survival advantage of about 3 months in advanced HCC patients [25]. Given the different modes of actions between the kinase inhibitor (sorafenib) and the cytotoxic agent (doxorubicin), it is perfectly reasonable to evaluate the combination of these two classes of drug and assess the clinical benefits that may bring to HCC patients. Excitingly, a recent phase II study revealed the combined use of sorafenib and doxorubicin (administrated as conventional TACE) in patients from the Asia-Pacific region with intermediate $\mathrm{HCC}$ [93]. The disease control rate and overall response rate were reported to be $91.2 \%$ and $52.4 \%$, respectively. Thus, concurrent sorafenib and doxorubicin-TACE therapy appears to be effective. Similar clinical studies are currently being conducted globally [94,95]. It is hoped that the findings from these studies corroborate the positive results obtained from the Asia-Pacific region [93].

Other molecularly targeted agents, such as Bevacizumab/Erlotinib, are currently under clinical investigation for therapy for HCC [96]. Bevacizumab is a humanized monoclonal antibody that inhibits vascular endothelial growth factor $A$, while Erlotinib is a reversible tyrosine kinase inhibitor that acts on the epidermal growth factor receptor [97]. As in the case of sorafenib, these drug actions are based on different mechanisms as compared with doxorubicin. It would be of great interest to evaluate their synergistic effects with doxorubicin and see if these might result in tumour shrinkage and enhanced survival.

\subsection{Combination with other treatment options}

Doxorubicin-TACE could offer the possibility of extending HCC patients' lives while waiting for curative treatment such as a liver transplant. It has been pointed out that the patient drop-off (e.g. death) risk while awaiting liver transplantation for HCC is $22 \%$ [98]. Doxorubicin-TACE treatment significantly reduced the drop-off risk, resulting in longer survival [98].

The combination of TACE with radiofrequency ablation (RFA) is a promising approach to treating HCC. RFA represents an attractive alternative to liver resection for patients with early-stage HCC. RFA uses radiofrequency energy-generated heat to destroy the tumour. The technique works well with small HCC $<$ $3 \mathrm{~cm}$ ), with an impressive 4-year survival rate of $66-82 \%$ as reported in Japan [99]. As the size of tumour grows beyond $5 \mathrm{~cm}$, the efficacy of RFA is generally reduced [100], probably due to increased blood flow leading to heat loss and/or incomplete ablation. It has been reported that by performing hepatic intraarterial embolization ahead of RFA treatment (TACE-RFA), the heat sink effect mediated by hepatic arterial flow should be minimized [101]. Giving TACE before RFA administration offers additional locoregional chemotherapy, which should result in better response and improved survival. Clinical studies have been conducted to evaluate the TACE-RFA procedure in HCC patients. Cheng et al. were among the first to report a clinical trial involving TACE-RFA in patients with HCC tumour sizes from 3 to $7.5 \mathrm{~cm}$ [102]. It has been shown that patients treated with TACE-RFA had better overall survival than those treated with TACE alone or RFA alone. A more recent study also revealed a similar picture [103]. With a median follow-up of 36 months, the 1-, 3-, and 4-year overall survival for the TACE-RFA treated patients and the RFA treated patients was $92.6 \%$ and $66.6 \%, 61.8 \%$ and $85.3 \%$, and $59 \%$ and $45.0 \%$, respectively. No significant difference in toxicity profiles was observed between the TACE-RFA treated patients and the RFA treated patients. 


\section{Conclusions}

HCC mortality is high, and the treatment options are limited. Surgical procedures such as liver resection or liver transplantation are still regarded as the only curative treatments currently available. In general, less than $20 \%$ of HCC patient are deemed suitable candidates to perform curative resection. TACE is emerging as a promising palliative treatment for HCC patients that are not suitable for surgical procedures. The recently developed DEB offers some operational convenience in performing the TACE procedure. Doxorubicin is one of the most commonly used cytotoxic drugs in TACE. Despite doxorubicin exhibiting a broad spectrum of anti-tumour activity, systemic administration can often lead to fatal cardiotoxicity and adverse side effects. As a liver directed, locoregional procedure, TACE considerably reduces the systemic exposure to doxorubicin, resulting in improved toxicity profiles, and has been shown to be useful in preventing tumour progression and in prolonging patients' lives. TACE in combination with targeted chemotherapy, such as sorafenib, has already demonstrated clinical advantages in the Asia-Pacific region. Moreover, concurrent use of TACE and RFA has shown promising survival benefits in several clinical studies. It is envisaged that doxorubicin administration via TACE could be one more useful tool in combatting HCC as it offers the exciting possibility of enhancing the currently available therapies for HCC patients. Obviously, more clinical studies are required to fully evaluate the clinical benefits of these combined approaches.

\section{References}

[1] GLOBOCAN 2008. International Agency for Cancer Research - Liver cancer fact sheet, http://globocan.iarc.fr/ (accessed 16 May, 2013)

[2] Hepatocellular carcinoma: ESMO Clinical Practice Guidelines for diagnosis, treatment and follow-up, European Society for Medical Oncology (2012).

[3] A. Forner, J.M. Llovet, J. Bruix, Lancet 31 (2012) 1245-1255.

[4] F.X. Bosch, J. Ribes, M. Díaz, R. Cléries, Gastroenterology 127 (2004) S5-S16.

[5] E.E. Calle, C. Rodriguez, K. Walker-Thurmond K, M.J. Thun, The New England Journal of Medicine 348 (2003) 1625-1638.

[6] J. Bruix, J.M. Llovet, Lancet 373 (2009) 614-616.

[7] S.C. Chuang, C. La Vecchia, P. Boffetta , Cancer Letters 286 (2009) 9-14.

[8] K. Yoshikawa, H. Kitaoka, The Japanese Journal of Surgery 1 (1971) 256-262.

[9] R.B. Weiss, Seminars in Oncology 19 (1992) 670-686.

[10] D.D. Von Hoff DD, M.W. Layard, P. Basa, H.L. Davis, A.L. Von Hoff, M. Rozencweig, F.M. Muggia, Annals of Internal Medicine 91 (1979) 710-717.

[11] B.I. Carr, S. Nagalla, in Hepatocellular Carcinoma: Diagnosis and Treatment, Brain Carr (Ed)., Humana Press, New York, USA, 2010, p. 527.

[12] M.C. Kew, H.A. Dos Santos, S. Sherlock, British Medical Journal 4 (1971) 408-411.

[13] A. Forner, A.J. Hessheimer, M. Isabel Real M, J. Bruix, Critical Reviews in Oncology/Hematology 60 (2006) 89-98.

[14] B.H. Zhang, B.H., Yang, Z.Y. Tang, Journal of Cancer Research and Clinical Oncology 130 (2004) 417422.

[15] T. Shuto, K. Hirohashi, S. Kubo, H. Tanaka, T. Tsukamoto, T. Yamamoto, T. Ikebe, H. Kinoshita, Surgery Today 28 (1998) 1124-1129 
[16] X.D. Zhou, Z.Y. Tang, B.H. Yang BH, Z.Y. Lin, Z.C. Ma, S.L. Ye, Z.Q. Wu, J. Fan, L.X. Qin, B.H. Zheng, Cancer 91:1479-1486

[17] V. Mazzaferro, E. Regalia, R. Doci, S. Andreola, A. Pulvirenti, F. Bozzetti, F. Montalto, M. Ammatuna, A. Morabito, L. Gennari, The New England Journal of Medicine 334 (1996) 693-699

[18] J.M. Llovet, J. Bruix, J. Fuster, J.C. Garcia-Valdecasas, L. Grande, A. Franca, C. Brú, M. Navasa, M.C. Ayuso, M. Solé, M.I. Real, R. Vilana, A. Rimola, J.Visa, J. Rodés, Hepatology 27 (1998) 1572-1577

[19] K. Ohnishi, H. Yoshioka, S. Ito, K. Fujiwara, Hepatology 27 (1998) 67-72.

[20] T. Shibata, Y. limuro, Y. Yamamoto, Y. Maetani, F. Ametani, K. Itoh, J. Konishi, Radiology 223 (2002) 331-337.

[21] R. Adam, E.J. Hagopian, M. Linhares, J. Krissat, E. Savier, D. Azoulay, F. Kunstlinger, D. Castaing, H. Bismuth, Archives of Surgery 137 (2002) 1332-1340.

[22] C.M. Pacella, G. Bizzarri, F. Magnolfi, P. Cecconi, B. Caspani, V. Anelli, A. Bianchini, D. Valle, S. Pacella, G. Manenti, Z. Rossi, Radiology 221 (2001) 712-720.

[23] P. Simmonds, British Medical Journal 321 (2000) 531-535.

[24] R.K. Kelley, A.P. Venook, Journal of Clinical Oncology, 26 (2008) 5845-5848.

[25] J.M. Llovet, S. Ricci, V. Mazzaferro, P. Hilgard, E. Gane, J.F. Blanc, A.C. de Oliveira, A. Santoro, J.L. Raoul, A. Forner, M. Schwartz, C. Porta, S. Zeuzem, L. Bolondi, T.F. Greten, P.R. Galle, J.F. Seitz, I. Borbath, D. Häussinger, T. Giannaris, M. Shan, M. Moscovici, D. Voliotis, J. Bruix, The New England Journal of Medicine 359 (2008) 378-390.

[26] A. Kennedy, S. Nag, R. Salem, R. Murthy, A.J. McEwan, C. Nutting, A. Benson, J. Espat, J.I. Bilbao, R.A. Sharma, J.P. Thomas, D. Coldwell, International Journal of Radiation Oncology, Biology and Physics 68 (2007) 13-23.

[27] D.L. Bartlett, J. Berlin, G.Y. Lauwers, W.A. Messersmith, N.J. Petrelli, A.P. Venook, Annals of Surgical Oncology 13 (2006) 1284-1292.

[28] J.W. Chung, Hepatogastroenterology 45 Suppl 3 (1998), 1236-1241.

[29] E. Liapi, K.H. Lee, C.C. Georgiades, K. Hong, J.F.H. Geschwind, Techniques in Vascular \& Interventional Radiology 10 (2007) 261-269.

[30] C. Breedis, G. Young, The American Journal of Pathology 30 (1954) 969-977.

[31] T. Nakashima, M. Kojiro, Seminars in Liver Disease 6 (1986) 259-266.

[32] S.W. Shin, Korean Journal of Radiology 10 (2009) 425-434.

[33] C.M. Lo, H. Ngan, W.K. Tso, C.L. Liu, C.M. Lam, R.T. Poon, S.T. Fan, J. Wong, Hepatology 35 (2002) 1164-1171.

[34] J.M. Llovet, M.I. Real, X. Montana, R. Planas, S. Coll, J. Aponte, C. Ayuso, M. Sala, J. Muchart, R. Sola, J. Rodes, J. Bruix, Lancet 359 (2002) 1734-1739.

[35] C. Camma, F. Schepis, A. Orlando, M. Albanese, L. Shahied, F. Trevisani, P. Andreone, A. Craxi, M. Cottone, Radiology 224 (2002) 47-54.

[36] J.M. Llovet, J. Bruix, Hepatology 37 (2003) 429-442.

[37] H. Nakamura, T. Hashimoto, H. Oi, S. Sawada, Radiology 170 (1989) 783-786.

[38] A.O. Chan, M.F. Yuen, C.K. Hui, W.K. Tso, C.L. Lai, Cancer 94 (2002) 1747-1752.

[39] J. Gao, F. Qian, A. Szymanski-Exner, N. Stowe, J. Haaga, Journal of Biomedical Materials Research 62 (2002) 308-314.

[40] K. Malagari, Expert Review of Anticancer Therapy 8 (2008) 1643-1650.

[41] M. Sadick, S. Haas, M. Loehr, M. Elshwi, M.V. Singer, J. Brade, S.O. Schoenberg, S.J. Diehl, Onkologie 33 (2010) 31-37.

[42] A.L. Lewis, M.W. Gonzalez, A.W. Lloyd, B. Hall, Y.Q. Tang, S.L. Willis, S.W. Leppard, L.C. Wolfenden, R.R. Palmer, P.W. Stratford, Journal of Vascular and Interventional Radiology 17 (2006) 335-342.

[43] J. Kettenbach, A. Stadler, I.V. Katzler, R. Schernthaner, M. Blum, J. Lammer, T. Rand, CardioVascular and Interventional Radiology 31 (2008) 468-476. 
[44] J. Lammer, K. Malagari, T. Vogl, F. Pilleul, A. Denys, A. Watkinson, M. Pitton, G. Sergent, T. Pfammatter, S. Terraz, Y. Benhamou, Y. Avajon, T. Gruenberger, M. Pomoni, H. Langenberger, M. Schuchmann, J. Dumortier, C. Mueller, P. Chevallier, R. Lencioni, P.V. Investigators, CardioVascular and Interventional Radiology 33 (2010) 41-52.

[45] K. Malagari, M. Pomoni, H. Moschouris, E. Bouma, J. Koskinas, A. Stefaniotou, A. Marinis, A. Kelekis, E. Alexopoulou, A. Chatziioannou, K. Chatzimichael, S. Dourakis, N. Kelekis, S. Rizos, D. Kelekis, CardioVascular and Interventional Radiology 35 (2012) 1119-1128.

[46] R. Yamada, M. Sato, M. Kawabata, H. Nakatsuka, K. Nakamura, S. Takashima, Radiology 148 (1983) 397-401.

[47] Doxorubicin Official FDA information, http://www.drugs.com/pro/doxorubicin.html (accessed 28 May, 2013).

[48] Lipodox Official FDA information, http://www.drugs.com/pro/lipodox.html (accessed 28 May, 2013).

[49] PSO 096-10 Doxorubicin hydrochloride (in heat sensitive liposomes), http://www.ema.europa.eu/docs/en GB/document library/Orphan designation/2011/03/WC500 102686.pdf (accessed 28 May, 2013).

[50] G. Powis, Pharmacology \& Therapeutics 35 (1987) 57-162.

[51] C.A. Frederick, L.D. Williams, G. Ughetto, G.A. van der Marel, J.H. van Boom, A Rich, A.H. Wang, Biochemistry 29 (1990) 2538-2549.

[52] F. Penault-Llorca, A. Cayre, F. Bouchet Mishellany, S. Amat, V. Feillel, G. Le Bouedec, J.P. Ferriere, M. De Latour, P. Chollet, International Journal of Oncology 22 (2003) 1319-1325;

[53] V. Stearns, B. Singh, T. Tsangaris, J.G. Crawford, A. Novielli, M.J. Ellis, C. Isaacs, M. Pennanen, C. Tibery, A. Farhad, R. Slack, D.F. Hayes, Clinical Cancer Research 9 (2003) 124-133.

[54] N.R. Bachur, S.L. Gordon, M.V. Gee, Molecular Pharmacology 13 (1977) 901-910.

[55] N.R. Bachur, M. Gee, Journal of Pharmacology and Experimental Therapeutics 197 (1976) 681-686.

[56] J. Cummings, L. Anderson, N Willmott, J.F. Smyth, European Journal of Cancer 27 (1991) 532-535.

[57] J.H. Doroshow, Cancer Research 43 (1983) 4543-4551.

[58] D.W. Reif, Free Radical Biology and Medicine 12 (1992) 417-427.

[59] D.A. Gewirtz, Biochemical Pharmacology 57 (1999) 727-741

[60] P.M. Kanter, H.S. Schwartz, Leukemia Research 3 (1979) 277-283.

[61] C.E. Myers, W.P. McGuire, R.H. Liss, I. Ifrim, K. Grotzinger, R.C. Young, Science 197 (1977) 165-167.

[62] D.H. Huffman, N.R. Bachur, Cancer Research 32 (1972) 600-605.

[63] N.K. Ahmed, R.L. Felsted, N.R Bachur, Journal of Pharmacology and Experimental Therapeutics 209 (1979) 12-19.

[64] K. Mross, P. Maessen, W.J. van der Vijgh, H. Gall, E. Boven, H.M. Pinedo, Journal of Clinical Oncology 6 (1988) 517-526.

[65] W.J. Pigram, W. Fuller, L.D. Hamilton, Nature 235 (1972) 17-19.

[66] E.N. Dessypris, D.E. Brenner, M.R. Baer, K.R. Hande, Cancer Research 48 (1988) 503-506.

[67] D.W. Yesair, P.S. Thayer, S. McNitt, K. Teague, European Journal of Cancer 16 (1980) 901-907.

[68] R.D. Olson, P.S. Mushlin, D.E. Brenner, S. Fleischer, B.J. Cusack, B.K. Chang, R.J. Boucek, Proceedings of the National Academy of Sciences 85 (1988) 3585-3589.

[69] P.K. Singal, T. Li, D. Kumar, I Danelisen, N. Iliskovic, Molecular and Cellular Biochemistry 207 (2000) 77-85.

[70] M.R. Bristow, P.D. Thompson, R.P. Martin, J.M. Mason, M.E. Billingham, D.C. Harrison, American Journal of Medicine 65 (1978) 823-832.

[71] K.J. Schimmel, D.J. Richel, R.B. van den Brink, H.J. Guchelaar, Cancer Treatment Review 30 (2004) 181-191. 
[72] S. Wang, S. Kotamraju, E. Konorev, S. Kalivendi, J. Joseph, B. Kalyanaraman, Biochemical Journal 367 (2002) 729-740.

[73] G. Minotti, Chemical Research in Toxicology 6 (1993) 134-146.

[74] G. Cairo, S. Recalcati, A. Pietrangelo, G. Minotti, Free Radical Biology and Medicine 32 (2002) 12371243.

[75] G. Minotti, A.F. Cavaliere, A. Mordente, M. Rossi, R. Schiavello, R. Zamparelli, G.F. Possati, Journal of Clinical Investigation 95 (1995) 1595-1605.

[76] P.M. Sokolove, M.B. Kester, J. Haynes, Biochemical Pharmacology, 46 (1993) 691-697.

[77] P.M. Sokolove, R.G. Shinaberry, Biochemical Pharmacology, 37 (1988) 803-812.

[78] T.V. Goolsby, F.A. Lombardo, Seminars in Oncology 33 (2006) 139-143.

[79] H.A. Burris, J. Hurtig, The Oncologist 15 (2010) 1227-1237.

[80] C.M. Camaggi, R. Comparsi, E. Strocchi, F. Testoni, B. Angelelli, F. Pannuti, Cancer Chemotherapy and Pharmacology 21 (1988) 221-228.

[81] S.C. Piscitelli, K.A. Rodvold, D.A. Rushing, D.A. Tewksbury, Clinical Pharmacology \& Therapeutics 53 (1993) 555-561.

[82] A. Gabizon, H. Shmeeda, Y. Barenholz, Clinical Pharmacokinetics 42 (2003) 419-436.

[83] S.N. Hilmer, V.C. Cogger, M. Muller, D.G. Le Couteur, Drug Metabolism and Disposition 32 (2004) 794-799.

[84] P.J. Johnson, C. Kalayci, N. Dobbs, N. Raby, E.M. Metivier, L. Summers, P. Harper, R. Williams, Journal of Hepatology 13 (1991) 120-127.

[85] M.V. Gonzalez, Y. Tang, G.J. Phillips, A.W. Lloyd, B. Hall, P.W. Stratford, A.L. Lewis, Journal of Materials Science Materials in Medicine 19 (2008) 767-775.

[86] K. Hong, A. Khwaja, E. Liapi, M.S. Torbenson, C.S. Georgiades, J.F. Geschwind, Clinical Cancer Research 12 (2006) 2563-2567.

[87] R.T.P. Poon, W.K. Tso, R.W.C. Pang, K.K.C. Ng, R. Woo, K.S. Tai, S.T. Fan, Clinical Gastroenterology and Hepatology 5 (2007) 1100-1108.

[88] M. Varela, M.I. Real, M. Burrel, A. Forner, M. Sala, M. Brunet, C. Ayuso, L. Castells, X. Montana, J.M. Llovet, J. Bruix, Journal of Hepatology 46 (2007) 474-481.

[89] J. Namur, S.J. Citron, M.T. Sellers, M.H. Dupuis, M. Wassef, M. Manfait, A. Laurent, Journal of Hepatology 55 (2011) 1332-1338.

[90] R. Lencioni, T. de Baere, M. Burrel, J.G. Caridi, J. Lammer, K. Malagari, R.C.G. Martin, E. O’Grady, M.I. Real, T.J. Vogl, A. Watkinson, J.F.H. Geschwind, CardioVascular and Interventional Radiology 35 (2012) 980-985.

[91] A. Fohlen, J.P. Pelage, in Transcatheter Embolization and Therapy, D. O. Kessel, C.E. Ray, Ed(s)., Springer, London, UK, 2010, p. 29.

[92] M. Brinckman, in Transcatheter Embolization and Therapy, D. O. Kessel, C.E. Ray, Ed(s)., Springer, London, UK, 2010, p. 41.

[93] Y.H. Chung, G. Han, J.H. Yoon, J. Yang, J. Wang, G.L. Shao, B.I. Kim, T.Y. Lee, Y. Chao, International Journal of Cancer 132 (2013) 2448-2458.

[94] Chemoembolization With or Without Sorafenib Tosylate in Treating Patients With Liver Cancer That Cannot Be Removed By Surgery, http://clinicaltrials.gov/ct2/show/NCT01004978 (accessed 30 May, 2013).

[95] Transarterial Chemoembolization Using Doxorubicin Beads With or Without Sorafenib Tosylate in Treating Patients With Liver Cancer That Cannot Be Removed By Surgery, http://clinicaltrials.gov/ct2/show/NCT01324076 (accessed 30 May, 2013).

[96] Erlotinib Plus Bevacizumab in Hepatocellular Carcinoma (HCC) as Second-line Therapy, http://clinicaltrials.gov/ct2/show/NCT01180959 (accessed 30 May, 2013). 
[97] S.C. Sweetman, (Ed)., Martindale: The Complete Drug Reference ( $35^{\text {th }}$ edition ed.), Pharmaceutical Press, London, UK, Electronic version, 2006.

[98] C. Frangakis, J.F. Geschwind, D. Kim, Y. Chen, A. Koteish, K. Hong, E. Liapi, C.S. Georgiades, CardioVascular and Interventional Radiology 34 (2011) 1254-1261.

[99] Y. Minami, M. Kudo, International Journal of Hepatology Article ID 104685 (2011) 9 pages.

[100] J. Huang, L. Yan, Z. Cheng, H. Wu, L. Du, J. Wang, Y. Xu, Y. Zeng, Annals of Surgery 252 (2010) 903912.

[101] T. Yamasaki, F. Kurokawa, H. Shirahashi, N. Kusano, K. Hironaka, K. Okita, Cancer 95 (2002) 23532360.

[102] B.Q. Cheng, C.Q. Jia, C.T. Liu, W. Fan, Q.L. Wang, Z.L. Zhang, C.H. Yi, Journal of the American Medical Association 299 (2008) 1669-1677.

[103] Z.W. Peng, Y.J. Zhang, M.S. Chen, L. Xu, H.H. Liang, X.J. Lin, R.P. Guo, Y.Q. Zhang, W.Y. Lau, Journal of Clinical Oncology 31 (2013) 426-432.

(C)2013 by the authors; licensee IAPC, Zagreb, Croatia. This article is an open-access article distributed under the terms and conditions of the Creative Commons Attribution license (http://creativecommons.org/licenses/by/3.0/) (cc) E EY 